\title{
On $(h, k)$-Dichotomies for Nonautonomous Linear Difference Equations in Banach Spaces
}

\author{
Mihai Gabriel Babuţia, ${ }^{1}$ Mihail Megan,, ${ }^{1,2}$ and Ioan-Lucian Popa ${ }^{1}$ \\ ${ }^{1}$ Department of Mathematics, Faculty of Mathematics and Computer Science, West University of Timişoara, \\ V. Pârvan Boulevard. no. 4, 300223 Timişoara, Romania \\ ${ }^{2}$ Academy of Romanian Scientists, Independenţei 54, 050094 Bucharest, Romania
}

Correspondence should be addressed to Mihail Megan; megan@math.uvt.ro

Received 30 April 2013; Revised 2 August 2013; Accepted 5 August 2013

Academic Editor: Timothy R. Marchant

\begin{abstract}
Copyright (C) 2013 Mihai Gabriel Babuţia et al. This is an open access article distributed under the Creative Commons Attribution License, which permits unrestricted use, distribution, and reproduction in any medium, provided the original work is properly cited.

This paper considers two general concepts of dichotomy for noninvertible and nonautonomous linear discrete-time systems in Banach spaces. These concepts use two types of dichotomy projections sequences (invariant and strongly invariant) and generalize some well-known dichotomy concepts (uniform, nonuniform, exponential, and polynomial). In the particular case of strongly invariant dichotomy projections, we present characterizations of these sequences and connections with other dichotomy concepts existent in the literature. Some illustrative examples clarify the implications between these concepts.
\end{abstract}

\section{Introduction}

In the last few years, the theory of difference equations has witnessed an impressive development. The notion of (uniform) exponential dichotomy introduced by Perron in [1] for differential equations and by Li in [2] for difference equations plays a central role in a substantial part of the theory of differential equations and dynamical systems.

The notion of dichotomy for differential equations has gained prominence since the appearance of two fundamental monographs of Massera and Schäffer [3] and Daleckii and Krein [4]. These were followed by the important book of Coppel [5] who synthesized and improved the results that existed in the literature up to 1978. Early works in the counterpart results of difference equations appeared in the paper of Coffman and Schäffer [6] and later, in 1981, when Henry included discrete dichotomies in his book [7]. This was followed by the classical monographs due to Agarwal [8] where ordinary and exponential dichotomy properties of difference equations are studied and various applications are provided. Significant work was reported by Pötzsche in [9].

One important and useful concept of dichotomy in the study of difference equations is the so-called (uniform) $(h, k)$ dichotomy concept introduced by Pinto in [10]. Since then, this concept has been extensively studied and applied; see, for example, Naulin and Pinto [11], Megan [12], Fenner [13], and Lin [14] where several examples are presented.

A new notion called nonuniform $(\mu, \nu)$-dichotomy is proposed by Bento and Silva in [15] for the continuous case and in $[16,17]$ for discrete time settings, for invertible systems, with growth rates given by increasing functions (sequences) which go to infinity. In the last years, this subject (resp., nonuniform exponential dichotomy and nonuniform polynomial dichotomy) became one of the subjects of large interest, significant results being obtained (see, e.g., [18-24]).

On the other hand, we can consider the case of noninvertible systems. From this point of view the paper of Aulbach and Kalkbrenner [25] is of interest, where the notion of exponential forward splitting is introduced, motivated by the fact that there are equations whose backward solutions are not guaranteed to exist. Indeed, this study is of interest in applications; see, for example, random dynamical systems, generated by random parabolic equations, which are not invertible (for more details, see Zhou et al. [26]).

Motivated by the above studies, in this paper, we consider two concepts of nonuniform $(h, k)$-dichotomies for difference equations in Banach spaces. These concepts use two concepts 
of sequences of projections: invariant and strongly invariant for the respective difference systems. Thus, we offer characterizations for these concepts and present connections between them. It is worth to mention the fact that if a sequence of projections is invariant for a reversible system then it is also strongly invariant for that system, and in this way the concepts merge. In this way, our study is related to the case of noninvertible systems.

\section{Preliminaries}

Let $X$ be a Banach space and $\mathscr{B}(X)$ the Banach space of all bounded linear operators on $X$. The norms on $X$ and on $\mathscr{B}(X)$ will be denoted by $\|\cdot\|$. The identity operator on $X$ is denoted by $I$. If $T \in \mathscr{B}(X)$, then we will denote by $\operatorname{Ker} T$ the kernel of $T$; that is,

$$
\begin{aligned}
& \text { Ker } T=\{x \in X \text { with } T x=0\}, \\
& \text { Range } T=\{T x \text {; with } x \in X\},
\end{aligned}
$$

respectively. We also denote by $\Delta$ the set of all pairs of all natural numbers $(m, n)$ with $m \geq n$; that is, $\Delta=\{(m, n) \in$ $\mathbb{N}^{2}$ with $\left.m \geq n\right\}$.

We consider the linear difference system

$$
x_{n+1}=A_{n} x_{n}
$$

where $A: \mathbb{N} \rightarrow \mathscr{B}(X)$ is a given sequence. For $(m, n) \in \Delta$, we define

$$
A_{m}^{n}= \begin{cases}A_{m-1} \cdot \ldots \cdot A_{n}, & \text { if } m>n \\ I, & \text { if } m=n .\end{cases}
$$

It is obvious that

$$
A_{m}^{n} A_{n}^{p}=A_{m}^{p}, \quad \forall(m, n),(n, p) \in \Delta,
$$

and every solution of $(\mathfrak{A})$ satisfies

$$
x_{m}=A_{m}^{n} x_{n} \quad \forall(m, n) \in \Delta .
$$

If for every $n \in \mathbb{N}$ the operator $A_{n}$ is invertible, then the system $(\mathfrak{A})$ is called reversible.

Definition 1. A sequence $P: \mathbb{N} \rightarrow \mathscr{B}(X)$ is called a sequence of projections if

$$
P_{n}^{2}=P_{n}, \quad \text { for every } n \in \mathbb{N} .
$$

Remark 2. If $P$ is a sequence of projections, then $Q=$ $I-P$ is also a sequence of projections (which is called the complementary sequence of projections of $P$ ) with $\operatorname{Ker} Q_{n}=$ Range $P_{n}$ and Range $Q_{n}=\operatorname{Ker} P_{n}$ for every $n \in \mathbb{N}$.

\section{3. $(h, k)$-Dichotomy with Invariant Projections}

Definition 3. A projection sequence $P$ is called invariant for the system $(\mathfrak{A})$ if

$$
A_{n} P_{n}=P_{n+1} A_{n}, \quad \forall n \in \mathbb{N} .
$$

Remark 4. If $P$ is invariant for ( $\mathfrak{U})$, then its complementary $Q$ is also invariant for $(\mathfrak{A})$. Furthermore, as a consequence of (7), we have

$$
A_{m}^{n} P_{n}=P_{m} A_{m}^{n}, \quad A_{m}^{n} Q_{n}=Q_{m} A_{m}^{n}
$$

for all $(m, n) \in \Delta$.

Remark 5. If $P$ is invariant for $(\mathfrak{U})$, then

(i) $A_{n}\left(\operatorname{Ker} P_{n}\right) \subset \operatorname{Ker} P_{n+1}$ for all $n \in \mathbb{N}$.

(ii) $A_{m}^{n}\left(\operatorname{Ker} P_{n}\right) \subset \operatorname{Ker} P_{m}$ and $A_{m}^{n}\left(\right.$ Range $\left.P_{n}\right) \subset$ Range $P_{m}$ for all $(m, n) \in \Delta$.

Definition 6. An nondecreasing sequence $\varphi: \mathbb{N} \rightarrow[1,+\infty)$ is said to be a growth rate sequence if $\varphi_{0}=1$ and $\lim _{n \rightarrow+\infty} \varphi_{n}=$ $+\infty$.

Let $h, k: \mathbb{N} \rightarrow[1,+\infty)$ be two growth rate sequences.

Definition 7. We say that the linear difference system $(\mathfrak{\mathcal { U }})$ admits a strong $(h, k)$-dichotomy if there exist a sequence of projections $P$ invariant for $(\mathfrak{A})$ and constants $N \geq 1, \alpha>0$, and $\beta \geq 0$ such that

$$
\begin{gathered}
h_{m}^{\alpha}\left\|A_{m}^{n} P_{n} x\right\| \leq N h_{n}^{\alpha} k_{n}^{\beta}\left\|P_{n} x\right\| \\
h_{m}^{\alpha}\left\|Q_{n} x\right\| \leq N h_{n}^{\alpha} k_{m}^{\beta}\left\|A_{m}^{n} Q_{n} x\right\|
\end{gathered}
$$

for all $(m, n, x) \in \Delta \times X$.

As particular cases of $(h, k)$-dichotomy, we have

(1) If $h_{n}=k_{n}=e^{n}$, then we say that ( $\left.\mathfrak{\mathcal { U }}\right)$ admits a strong exponential dichotomy.

(2) If $h_{n}=e^{n}$ and $\beta=0$, then we say that $\operatorname{system}(\mathfrak{A})$ admits a strong uniform exponential dichotomy.

(3) If $h_{n}=k_{n}=n+1$, then we say that system ( $\left.\mathfrak{\mathcal { I }}\right)$ admits a strong polynomial dichotomy.

(4) If $h_{n}=n+1$ and $\beta=0$, then we say that system ( $\left.\mathfrak{U}\right)$ admits a strong uniform polynomial dichotomy.

Remark 8. If the linear difference system $(\mathfrak{\mathfrak { U }})$ admits a strong $(h, k)$-dichotomy, then for all $(m, n) \in \Delta$ the following assertion holds:

$$
A_{m}^{n} Q_{n} x=0 \Longrightarrow Q_{n} x=0 .
$$

Definition 9. We say that the linear difference system ( $\mathfrak{\mathcal { I }})$ admits an $(h, k)$ dichotomy if there exist a sequence of projections $P$ invariant for $(\mathfrak{U})$ and constants $N \geq 1, \alpha>0$, and $\beta \geq 0$ such that

$$
\begin{gathered}
h_{m}^{\alpha}\left\|A_{m}^{n} P_{n}\right\| \leq N h_{n}^{\alpha} k_{n}^{\beta} \\
h_{m}^{\alpha} \leq N h_{n}^{\alpha} k_{m}^{\beta}\left\|A_{m}^{n} Q_{n}\right\|
\end{gathered}
$$

for all $(m, n, x) \in \Delta \times X$.

If the system $(\mathfrak{U})$ admits a $(h, k)$-dichotomy and we take into account the particular cases from above, we obtain the notions of exponential dichotomy, uniform exponential 
dichotomy, polynomial dichotomy, and uniform polynomial dichotomy, respectively.

From the previous definition, we have that

$$
\left\|P_{n}\right\| \leq N k_{n}^{\beta}, \quad \forall n \in \mathbb{N} .
$$

Definition 10. If $k: \mathbb{N} \rightarrow[1,+\infty)$ is a given growth rate sequence and $P$ is a sequence of projections that verifies (14), then we say that $P$ is $k$-bounded. ing.

As particular cases of $k$-boundedness, we have the follow-

(i) If there exist $M \geq 1$ and $\gamma \geq 0$ such that $\|P(n)\| \leq$ $M e^{\gamma n}$ for all $n \in \mathbb{N}$, then we say that $P$ is exponentially bounded.

(ii) If there exist $M \geq 1$ and $\gamma \geq 0$ such that $\|P(n)\| \leq$ $M(n+1)^{\gamma}$ for all $n \in \mathbb{N}$, then we say that $P$ is polynomially bounded.

(iii) If there exist $M \geq 1$ such that $\|P(n)\| \leq M$ for all $n \in \mathbb{N}$, then we say that $P$ is bounded.

Remark 11. It is worth to mention that the concept that we name "strong $(h, k)$-dichotomy," in the exponential case with bounded sequences of projections, is in fact the notion of exponential forward splitting considered in [25, Definition 2.1]. The notion of strong (uniform) exponential dichotomy has been also considered in [27]. The name of "strong dichotomy" derives from the fact that it implies the other dichotomy concept in this paper (under the $k$-boundedness assumption of the sequence of the projections). It is important to state that the "strong dichotomy" from this paper differs from the "strong dichotomy" defined in [18].

Remark 12. Through the following two examples we will show that for a difference system $(\mathfrak{\mathfrak { A }})$ the concepts of strong $(h, k)$ dichotomy and $(h, k)$-dichotomy are distinct, neither of them implying the other one.

Example 13. Consider $X=l^{1}$ the Banach space of all real valued sequences $x=\left(x_{n}\right)_{n \geq 0}$ endowed with the norm $\|x\|_{1}=$ $\sum_{j=0}^{+\infty}\left|x_{j}\right|$. Fix two growth rate sequences $h, k: \mathbb{N} \rightarrow[1,+\infty)$. Define, for every $n \in \mathbb{N}, P_{n}: l^{1} \rightarrow l^{1}$ by $P_{n} x=\left(y_{j}^{n}\right)_{j \geq 0}$ given by

$$
y_{j}^{n}= \begin{cases}x_{j}+k_{n}^{k_{n}} \cdot x_{j+1}, & \text { if } j \text { is even, } \\ 0, & \text { if } j \text { is odd. }\end{cases}
$$

One can see that $P_{n} \in \mathscr{B}\left(l^{1}\right)$ for all $n \in \mathbb{N}, P$ is a sequence of projections on $l^{1}$ and if we pick $x \in l^{1}$ satisfying $x_{2 j+1}=$ $1 /(j+1)^{2}$ and $x_{2 j}=0$, for all $j \in \mathbb{N}$, a simple computation gives us that

$$
\left\|P_{n} x\right\|_{1}=k_{n}^{k_{n}}\|x\|_{1}
$$

from where we deduce that

$$
\left\|P_{n}\right\| \geq k_{n}^{k_{n}}, \quad \forall n \in \mathbb{N} .
$$

In addition, we have the following properties:

$$
P_{m} P_{n}=P_{n}, \quad P_{n} P_{m}=P_{m}, \quad\left\|Q_{m} x\right\|_{1} \geq\left\|Q_{n} x\right\|_{1}
$$

for all $(m, n, x) \in \Delta \times l^{1}$.

Consider the linear difference system

$$
x_{n+1}=\frac{h_{n}}{h_{n+1}} P_{n} x_{n}+\frac{h_{n+1}}{h_{n}} Q_{n+1} x_{n},
$$

where $Q: \mathbb{N} \rightarrow \mathscr{B}\left(l^{1}\right)$ is the complementary sequence of projections of $P$.

Some computations show us that $A_{m}^{n}$ is given by

$$
A_{m}^{n}=\frac{h_{n}}{h_{m}} P_{n}+\frac{h_{m}}{h_{n}} Q_{m}
$$

for all $(m, n) \in \Delta$.

One can check that the sequence of projections is invariant for the system $(\mathfrak{U})$. From the fact that

$$
\begin{gathered}
h_{m}\left\|A_{m}^{n} P_{n} x\right\|_{1}=h_{n}\left\|P_{n} x\right\|_{1} \leq h_{n} k_{n}\left\|P_{n} x\right\|_{1}, \\
h_{n} k_{m}\left\|A_{m}^{n} Q_{n} x\right\|_{1}=k_{m} h_{m}\left\|Q_{m} x\right\|_{1} \geq h_{m}\left\|Q_{n} x\right\|_{1}
\end{gathered}
$$

for all $(m, n, x) \in \Delta \times l^{1}$, we have that the system $(\mathfrak{A})$ admits a strong $(h, k)$-dichotomy.

Indeed, assume that the system $(\mathfrak{U})$ admits a $(h, k)$ dichotomy. Then, by (14) and (17), we get the inequalities

$$
k_{n}^{k_{n}} \leq\left\|P_{n}\right\| \leq N k_{n}^{\beta} \quad \forall n \in \mathbb{N} .
$$

Because the sequence $k$ is a growth rate, choose $n_{\beta}$ such that

$$
k_{n} \geq \beta+1 \quad \forall n \geq n_{\beta} .
$$

Hence, the preceding inequality implies that

$$
k_{n} \leq k_{n}^{k_{n}-\beta} \leq N \quad \forall n \geq n_{\beta},
$$

which, by making $n \rightarrow \infty$, contradicts the fact that $k$ is a growth rate. Thus, we can see that the system $(\mathfrak{A})$ fails to admit a $(h, k)$-dichotomy.

Example 14. On $X=\mathbb{R}^{3}$ consider the sequence $A: \mathbb{N} \rightarrow$ $\mathscr{B}\left(\mathbb{R}^{3}\right)$ defined, for $n \in \mathbb{N}$ and $x=\left(x_{1}, x_{2}, x_{3}\right) \in \mathbb{R}^{3}$, by

$$
A_{n} x=\left(\frac{h_{n}}{h_{n+1}} x_{1}, \frac{h_{n+1}}{h_{n}} x_{2}, 0\right) .
$$

By considering the linear difference system $(\mathfrak{Y})$ associated to the above-defined sequence of bounded linear operators, we obtain that for all $(m, n, x) \in \Delta \times \mathbb{R}^{3}$ we have

$$
A_{m}^{n} x=\left(\frac{h_{n}}{h_{m}} x_{1}, \frac{h_{m}}{h_{n}} x_{2}, 0\right) .
$$

We define the sequence of projections $P: \mathbb{N} \rightarrow \mathscr{B}\left(\mathbb{R}^{3}\right)$ by

$$
P_{n}\left(x_{1}, x_{2}, x_{3}\right)=\left(x_{1}, 0,0\right)
$$


for all $n \in \mathbb{N}$ and $\left(x_{1}, x_{2}, x_{3}\right) \in \mathbb{R}^{3}$. One can see that $P$ is invariant for the system $(\mathfrak{A})$ and

$$
h_{m}\left\|A_{m}^{n} P_{n}\right\|=h_{n} \leq h_{n} k_{m}
$$

for all $(m, n) \in \Delta$. Moreover, for $(m, n) \in \Delta$, if we pick $x=$ $(0,1,0)$, then we get that

$$
h_{n}\left\|A_{m}^{n} Q_{n} x\right\|=h_{m}\|x\|,
$$

from where we obtain that

$$
h_{m} \leq h_{n}\left\|A_{m}^{n} Q_{n}\right\| \leq h_{n} k_{m}\left\|A_{m}^{n} Q_{n}\right\|
$$

for all $(m, n) \in \Delta$.

By (28) and (30), we conclude that the system ( $\mathfrak{A})$ admits a $(h, k)$-dichotomy.

Because $Q_{0}(0,0,1) \neq(0,0,0)$ and $A_{1}^{0}(0,0,1)=(0,0,0)$, by Remark 8 , it follows that the system $(\mathfrak{A})$ does not admit a strong $(h, k)$-dichotomy.

\section{4. $(h, k)$-Dichotomy with Strongly Invariant Projections}

Definition 15. A sequence of projections $P$ is called strongly invariant for the system $(\mathfrak{U})$ if $P$ is invariant for $(\mathfrak{A})$ and for all $n \in \mathbb{N}$ the restriction of $A_{n}$ to $\operatorname{Ker} P_{n}$ is an isomorphism from $\operatorname{Ker} P_{n}$ to $\operatorname{Ker} P_{n+1}$.

Remark 16. $P$ is strongly invariant for the system $(\mathfrak{A})$ if and only if for all $(m, n) \in \Delta$ the restriction of $A_{m}^{n}$ to $\operatorname{Ker} P_{n}$ is an isomorphism from $\operatorname{Ker} P_{n}$ to $\operatorname{Ker} P_{m}$.

Remark 17. In the exponential setting, under the hypotheses that $P$ is a strongly invariant for a system $(\mathfrak{A})$ and it is bounded, it can be easily checked that the concept of strong exponential dichotomy defined in our paper is in fact the concept of exponential splitting defined in [25, Definition 4.1] or the concept of exponential dichotomy from [28, Definition $1.1]$.

Remark 18. If the sequence of projections $P$ is invariant for the reversible system $(\mathfrak{A})$, then it is also strongly invariant for $(\mathfrak{A})$.

Indeed, if $P$ is invariant for the reversible system $(\mathfrak{A})$, then for every $y \in \operatorname{Ker} P_{n+1}$ we have $y=A_{n}\left(A_{n}\right)^{-1} y=A_{n} x$ with $x=\left(A_{n}\right)^{-1} y \in \operatorname{Ker} P_{n}$.

There are linear difference systems which admit $(h, k)$ dichotomy with respect to projection sequences which are invariant but are not strongly invariant for the system. This phenomenon is illustrated by the following example.

Example 19. On $X=\mathbb{R}^{3}$, with $\|x\|=\left|x_{1}\right|+\left|x_{2}\right|+\left|x_{3}\right|$, we consider the system generated by the sequence $A_{n}$ defined by

$$
A_{n}\left(x_{1}, x_{2}, x_{3}\right)=\left(\frac{x_{1}}{e}, e x_{2}, 0\right)
$$

for all $n \in \mathbb{N}$ and $x=\left(x_{1}, x_{2}, x_{3}\right) \in \mathbb{R}^{3}$. It is immediate to see that

$$
A_{m}^{n}= \begin{cases}\left(e^{n-m} x_{1}, e^{m-n} x_{2}, 0\right), & m \neq n, \\ I, & m=n .\end{cases}
$$

Define the sequence of projections $P: \mathbb{N} \rightarrow \mathscr{B}(X)$ by

$$
P_{n}\left(x_{1}, x_{2}, x_{3}\right)=\left(x_{1}, 0,0\right) \quad \forall n \in \mathbb{N},\left(x_{1}, x_{2}, x_{3}\right) \in \mathbb{R}^{3} .
$$

We have that the sequence $P$ is invariant for the system $(\mathfrak{A})$. Moreover, one can see that

$$
A_{m}^{n} P_{n} x=e^{n-m} P_{n} x
$$

and hence

$$
\left\|A_{m}^{n} P_{n}\right\|=e^{-(m-n)}\left\|P_{n}\right\| \quad \forall(m, n) \in \Delta .
$$

Moreover, for $(m, n) \in \Delta, m \neq n$, and $x_{0}=(0,1,0) \in \mathbb{R}^{3}$, we have that

$$
\left\|A_{m}^{n} Q_{n} x_{0}\right\|=e^{m-n}\left\|Q_{n} x_{0}\right\| .
$$

On the other hand, it is easy to check that

$$
A_{n}^{n} Q_{n} x_{0}=e^{n-n} Q_{n} x_{0} ;
$$

hence

$$
\left\|A_{m}^{n} Q_{n}\right\| \geq e^{m-n}\left\|Q_{n}\right\|=e^{m-n} \quad \forall(m, n) \in \Delta .
$$

This shows us that we have a system $(\mathfrak{A})$ which is (uniformly) exponentially dichotomic with the invariant projection sequence $P$. But $P$ is not strongly invariant because, for example, $A_{1} Q_{n}(0,0,1)=(0,0,0)$.

Remark 20. Our second concept of $(h, k)$-dichotomy introduced in this paper (see Definition 9) can be viewed in the light of the general dichotomies presented in [17], by making the choice $a(m, n)=(h(n) / h(m))^{\alpha} \cdot k(n)^{\beta}$ and $b(m, n)=(h(n) / h(m))^{\alpha} \cdot k(m)^{\beta}$ in the case of strongly invariant sequences of projections and by observing that the inequality (D2) from [17], namely, $\left\|\left(\left.A_{m}^{n}\right|_{\operatorname{Ker} P_{n}}\right)^{-1} Q_{m}\right\| \leq$ $b(m, n)$, implies the corresponding instability inequality from our dichotomy concept by observing that

$$
1 \leq\left\|\left.A_{m}^{n}\right|_{\operatorname{Ker} P_{n}}\left(\left.A_{m}^{n}\right|_{\operatorname{Ker} P_{n}}\right)^{-1} Q_{m}\right\| \leq\left\|\left.A_{m}^{n}\right|_{\operatorname{Ker} P_{n}}\right\| \cdot b(m, n) .
$$

Moreover, under the assumption of $k$-boundedness of the sequence of the projections and strong invariance, the notion of strong $(h, k)$-dichotomy from our paper is a particular case of the general dichotomies from [17], by making the same choice of the sequences $a(m, n)$ and $b(m, n)$.

The above-mentioned concept is useful in input-output techniques, and we mention the work of B. Sasu and A. L. Sasu [29], where, in the uniform exponential setting, from a sufficient condition of admissibility of pairs of function spaces to an associated control system of $(\mathfrak{\mathcal { U }})$, they deduce the property of uniform exponential dichotomy and the boundedness of the sequence of projections.

Even in the particular case of exponential dichotomy, the implication that gives (39) from the preceding remark fails to hold, showing that there exist reversible systems that verify Definition 9 and do not satisfy the dichotomic behavior pointed out by (39), even in the case of bounded sequences of projections. 
Remark 21. If the sequence of projections $P$ is strongly invariant for the system $(\mathfrak{\mathfrak { U }})$, then

(i) for every $n \in \mathbb{N}$ there is an isomorphism $B_{n}$ from the $\operatorname{Ker} P_{n+1}$ to $\operatorname{Ker} P_{n}$ such that $A_{n} B_{n} Q_{n+1}=$ $Q_{n}$ and $B_{n} A_{n} Q_{n}=Q_{n} ;$

(ii) for all $(m, n) \in \Delta$ there is an isomorphism $B_{m}^{n}$ from $\operatorname{Ker} P_{m}$ to $\operatorname{Ker} P_{n}$ with

$$
\begin{aligned}
A_{m}^{n} B_{m}^{n} Q_{m} & =Q_{m}, \\
B_{m}^{n} A_{m}^{n} Q_{n} & =Q_{n}
\end{aligned}
$$

for all $(m, n) \in \Delta$.

In particular case when $(\mathfrak{A})$ is invertible and $P$ is invariant for $(\mathfrak{U})$, then $B_{n}=\left(A_{n}\right)^{-1}$ for every $n \in \mathbb{N}$ and $B_{m}^{n}=\left(A_{m}^{n}\right)^{-1}$ for all $(m, n) \in \Delta$.

Proposition 22. Let $h$ and $k$ be growth rate sequences and $P$ a sequence of projections that is strongly invariant for a system $(\mathfrak{A})$. Then the system $(\mathfrak{U})$ admits a strong $(h, k)$-dichotomy if and only if there exist $N \geq 1, \alpha>0$, and $\beta \geq 0$ such that

$$
\begin{aligned}
& h_{m}^{\alpha}\left\|A_{m}^{n} P_{n} x\right\| \leq N h_{n}^{\alpha} k_{n}^{\beta}\left\|P_{n} x\right\|, \\
& h_{m}^{\alpha}\left\|B_{m}^{n} Q_{m} x\right\| \leq N h_{n}^{\alpha} k_{m}^{\beta}\left\|Q_{m} x\right\|
\end{aligned}
$$

for all $(m, n, x) \in \Delta \times X$, where $B_{m}^{n}$ are the images of the function $B$ defined in Remark 21.

Proof. We have to prove the equivalence of (10) and (43). Let $(m, n, x) \in \Delta \times X$. For the necessity, by (40), we have

$$
h_{m}^{\alpha}\left\|B_{m}^{n} Q_{m} x\right\| \leq N h_{n}^{\alpha} k_{m}^{\beta}\left\|A_{m}^{n} B_{m}^{n} Q_{m} x\right\|=N h_{n}^{\alpha} k_{m}^{\beta}\left\|Q_{m} x\right\| .
$$

For the sufficiency, by (41), we have

$$
\begin{aligned}
h_{m}^{\alpha}\left\|Q_{n} x\right\| & =h_{m}^{\alpha}\left\|B_{m}^{n} Q_{m} A_{m}^{n} Q_{n} x\right\| \\
& \leq N h_{n}^{\alpha} k_{m}^{\beta}\left\|Q_{m} A_{m}^{n} Q_{n} x\right\|=N h_{n}^{\alpha} k_{m}^{\beta}\left\|A_{m}^{n} Q_{n} x\right\|
\end{aligned}
$$

which concludes our proof.

Proposition 23. Let $h$ and $k$ be growth rate sequences and $P$ a sequence of projections that is strongly invariant for a system ( $\mathfrak{A})$. If there exist $N \geq 1, \alpha>0$, and $\beta \geq 0$ such that the system ( $\mathfrak{A})$ has the properties

$$
\begin{aligned}
& h_{m}^{\alpha}\left\|A_{m}^{n} P_{n}\right\| \leq N h_{n}^{\alpha} k_{n}^{\beta}, \\
& h_{m}^{\alpha}\left\|B_{m}^{n} Q_{m}\right\| \leq N h_{n}^{\alpha} k_{m}^{\beta}
\end{aligned}
$$

for all $(m, n) \in \Delta$, where $B_{m}^{n}$ are the images of the function $B$ defined in Remark 21. Then, $(\mathfrak{U})$ admits a $(h, k)$-dichotomy.

Proof. We have to show that (47) implies (13). Let $(m, n) \in \Delta$. The conclusion follows from

$$
\begin{aligned}
h_{m}^{\alpha} & \leq h_{m}^{\alpha}\left\|Q_{n}\right\|=h_{m}^{\alpha}\left\|B_{m}^{n} Q_{m} A_{m}^{n} Q_{n}\right\| \\
& \leq h_{m}^{\alpha}\left\|B_{m}^{n} Q_{m}\right\|\left\|A_{m}^{n} Q_{n}\right\| \leq N h_{n}^{\alpha} k_{m}^{\beta}\left\|A_{m}^{n} Q_{n}\right\| .
\end{aligned}
$$

The converse of the preceding proposition is not generally valid, as shown in the following example.

Example 24. On $X=l^{1}$, consider $\left(k_{n}\right)$ an arbitrary growth rate, $h_{n}=e^{n}$ for $n \in \mathbb{N}, \alpha=1$ and $\beta=0$. Define the sequence $A: \mathbb{N} \rightarrow \mathscr{B}\left(l^{1}\right)$ by $A_{n} x=\left(y_{j}(n)\right)_{j \geq 0}$, where

$$
\begin{array}{r}
y_{3 j+1}(n)=\frac{1}{e} \cdot x_{3 j+1} \\
y_{3 j+2}(n)=e \cdot x_{3 j+2} \\
y_{3 j}(n)=e^{e^{n}-e^{n+1}} \cdot x_{3 j} \\
\quad j \in \mathbb{N} .
\end{array}
$$

Then, we have that for $(m, n, x) \in \Delta \times l^{1}, A_{m}^{n} x=\left(y_{j}(m, n)\right)_{j \geq 0}$ with

$$
\begin{array}{r}
y_{3 j+1}(m, n)=e^{n-m} \cdot x_{3 j+1} \\
y_{3 j+2}(m, n)=e^{m-n} \cdot x_{3 j+2} \\
y_{3 j}(m, n)=e^{e^{n}-e^{m}} \cdot x_{3 j} \\
j \in \mathbb{N} .
\end{array}
$$

Define, for every $n \in \mathbb{N}$, the projection $P_{n}: l^{1} \rightarrow l^{1}$ by $P_{n} x=$ $\left(a_{j}\right)_{j \geq 0}$ where

$$
\begin{array}{r}
a_{3 j+1}=x_{3 j+1} \\
a_{3 j+2}=0 \\
a_{3 j}=0 \\
j \in \mathbb{N} .
\end{array}
$$

It is easy to check that the sequence of projections $P$ is strongly invariant for the system $(\mathfrak{U})$, and for $(m, n, x) \in \Delta \times l^{1}$, $B_{m}^{n} Q_{m} x=\left(z_{j}(m, n)\right)_{j \geq 0}$ is given by

$$
\begin{array}{r}
z_{3 j+1}(m, n)=0 \\
z_{3 j+2}(m, n)=e^{n-m} \cdot x_{3 j+2} \\
z_{3 j}(m, n)=e^{e^{m}-e^{n}} \cdot x_{3 j} \\
j \in \mathbb{N} .
\end{array}
$$

A simple computation shows us that

$$
e^{m}\left\|A_{m}^{n} P_{n}\right\|=e^{n}
$$

for all $(m, n) \in \Delta$ and by choosing $x \in l^{1}$ with $x_{3 j+1}=x_{3 j}=0$ and $x_{3 j+2}=1 /(j+1)^{2}$, it follows that

$$
e^{m} \leq\left\|A_{m}^{n} Q_{n}\right\| e^{n} .
$$

Hence, the system $(\mathfrak{A})$ admits a $(h, k)$-dichotomy. 
Assume now that there exist $\alpha>0, N \geq 1$, and $\beta \geq 0$ with

$$
h_{m}^{\alpha}\left\|B_{m}^{n} Q_{m}\right\| \leq N h_{n}^{\alpha} k_{m}^{\beta}
$$

for all $(m, n) \in \Delta$. In our case, the preceding inequality becomes

$$
e^{\alpha m}\left\|B_{m}^{n} Q_{m}\right\| \leq N e^{\alpha n}
$$

for all $(m, n) \in \Delta$.

Consider that $n=0, m \in \mathbb{N}$, and $x \in l^{1}$ given by

$$
\begin{array}{r}
x_{3 j+1}=x_{3 j+2}=0 \\
x_{3 j}=\frac{1}{(j+1)^{2}} \\
j \in \mathbb{N} .
\end{array}
$$

It follows that

$$
\left\|B_{m}^{0} Q_{m} x\right\|_{1}=e^{e^{m}-1} \cdot \frac{\pi}{\sqrt{6}}=e^{e^{m}-1}\|x\|_{1},
$$

from where we get that

$$
\left\|B_{m}^{0} Q_{m}\right\| \geq e^{e^{m}-1} .
$$

Combining the above inequality with (56), we get that

$$
e^{e^{m}+\alpha m-1} \leq N
$$

for all $m \in \mathbb{N}$, which is a contradiction.

Moreover, the converse of the implication from Proposition 23 does not hold not even in the finite dimensional case, as we can see from the following example.

Example 25. Consider that $h_{n}=k_{n}=e^{n}, n \in \mathbb{N}$. Consider, on $X=\mathbb{R}^{3}$, the sequence $A: \mathbb{N} \rightarrow \mathscr{B}\left(\mathbb{R}^{3}\right)$ is defined by

$$
A_{n}\left(x_{1}, x_{2}, x_{3}\right)=\left(\frac{x_{1}}{e}, e x_{2}, \frac{x_{3}}{e^{2}}\right) .
$$

A simple computation shows us that

$$
A_{m}^{n}\left(x_{1}, x_{2}, x_{3}\right)=\left(e^{n-m} x_{1}, e^{m-n} x_{2}, e^{n^{2}-m^{2}} x_{3}\right)
$$

for all $(m, n) \in \Delta$ and $\left(x_{1}, x_{2}, x_{3}\right) \in \mathbb{R}^{3}$. Define the (constant) sequence of projections $P: \mathbb{N} \rightarrow \mathscr{B}(X)$ by

$$
P_{n}\left(x_{1}, x_{2}, x_{3}\right)=\left(x_{1}, 0,0\right) \quad \forall n \in \mathbb{N},\left(x_{1}, x_{2}, x_{3}\right) \in \mathbb{R}^{3} .
$$

We have that $P$ is strongly invariant to the system $(\mathfrak{A})$ with

$$
\left(\left.A_{m}^{n}\right|_{\operatorname{Ker} P_{n}}\right)^{-1}\left(0, x_{2}, x_{3}\right)=\left(0, e^{n-m} x_{2}, e^{m^{2}-n^{2}} x_{3}\right)
$$

for all $(m, n) \in \Delta$ and for all $\left(0, x_{2}, x_{3}\right) \in \operatorname{Ker} P_{n}$. A straightforward computation shows us that

$$
\left\|A_{m}^{n} P_{n}\right\|=e^{-(m-n)}, \quad\left\|A_{m}^{n} Q_{n}\right\| \geq e^{m-n}
$$

for all $(m, n) \in \Delta$; hence, $(\mathfrak{A})$ satisfies the conditions from Definition 9 (in the exponential case).

Assume by a contradiction that there exist $N \geq 1, \alpha>0$, and $\beta \geq 0$ such that

$$
\begin{gathered}
\left\|A_{m}^{n} P_{n}\right\| \leq N e^{\beta n} e^{-\alpha(m-n)} \\
\left\|\left.\left(A_{m}^{n}\right)\right|_{\text {Ker } P_{n}} ^{-1}\right\| \leq N e^{\beta m} e^{-\alpha(m-n)}
\end{gathered}
$$

for all $(m, n) \in \Delta$. Choose $n=0$. From the fact that

$$
\left\|\left(\left.A_{m}^{0}\right|_{\operatorname{Ker} P_{0}}\right)^{-1}\right\|=e^{m^{2}} \quad \forall m \in \mathbb{N} .
$$

From (67) and (68), we get the contradiction

$$
e^{m^{2}} \leq N e^{(\beta-\alpha) m} \quad \forall m \in \mathbb{N} .
$$

Proposition 26. Let $h$ and $k$ be growth rate sequences and $P$ a sequence of projections that is strongly invariant for a system ( $\mathfrak{A})$. If there exist $N \geq 1, \alpha>0$, and $\beta \geq 0$ such that the

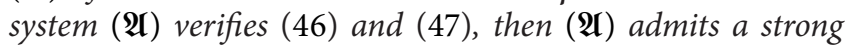
$(h, k)$-dichotomy.

Proof. Let $(m, n, x) \in \Delta \times X$. It follows that

$$
\begin{gathered}
h_{m}^{\alpha}\left\|A_{m}^{n} P_{n} x\right\| \leq h_{m}^{\alpha}\left\|A_{m}^{n} P_{n}\right\|\left\|P_{n} x\right\| \leq N h_{n}^{\alpha} k_{n}^{\beta}\left\|P_{n} x\right\|, \\
h_{m}^{\alpha}\left\|B_{m}^{n} Q_{m} x\right\| \leq h_{m}^{\alpha}\left\|B_{m}^{n} Q_{m}\right\|\left\|Q_{m} x\right\| \leq N h_{n}^{\alpha} k_{m}^{\beta}\left\|Q_{m} x\right\|
\end{gathered}
$$

which, by Proposition 22, concludes the proof.

Theorem 27. Let $h$ and $k$ be growth rates and $P$ a sequence of projections that is strongly invariant for a system $(\mathfrak{\mathfrak { I }})$. Then, the system $(\mathfrak{\mathfrak { U }})$ verifies (46) and (47) if and only if $(\mathfrak{\mathfrak { U }})$ admits a strong $(h, k)$-dichotomy and $P$ is $k$-bounded.

Proof. The necessity follows from Proposition 26 and (14).

For the sufficiency, let $M \geq 1$ and $\gamma \geq 0$ such that

$$
\left\|P_{n}\right\| \leq M k_{n}^{\gamma}, \quad \forall n \in \mathbb{N} .
$$

Let $(m, n, x) \in \Delta \times X$. From

$$
\begin{gathered}
h_{m}^{\alpha}\left\|A_{m}^{n} P_{n} x\right\| \leq N h_{n}^{\alpha} k_{n}^{\beta}\left\|P_{n} x\right\| \leq 2 M N h_{n}^{\alpha} k_{n}^{\beta+\gamma}\|x\|, \\
h_{m}^{\alpha}\left\|B_{m}^{n} Q_{m} x\right\| \leq N h_{n}^{\alpha} k_{m}^{\beta}\left\|Q_{m} x\right\| \leq 2 M N h_{n}^{\alpha} k_{m}^{\beta+\gamma}\|x\|,
\end{gathered}
$$

the conclusion follows.

Corollary 28. Let $P$ be a sequence of projections that is strongly invariant for a system $(\mathfrak{\mathfrak { U }})$.

(i) If $h_{n}=k_{n}=e^{n}$, then the system ( $\left.\mathfrak{A}\right)$ verifies (46) and (47) if and only if it is exponentially dichotomic and $P$ is exponentially bounded.

(ii) If $h_{n}=k_{n}=n+1$, then the system ( $\left.\mathfrak{A}\right)$ verifies (46) and (47) if and only if it is polynomially dichotomic and $P$ is polynomially bounded.

(iii) If $h_{n}=e^{n}$ (resp., $h_{n}=n+1$ ) and $\beta=0$, then the system ( $\mathfrak{A})$ verifies (46) and (47) if and only if it is uniformly exponentially (polynomially) dichotomic and $P$ is bounded. 


\section{Acknowledgments}

The authors would like to thank the referees for carefully reading the paper and for their valuable suggestions and comments, which led to the current improved form of the paper. Mihai Gabriel Babuţia is supported by a grant of the Romanian National Authority for Scientific Research, CNCS UEFISCDI, Project no. PN-II-RU-TE-2011-3-0103.

\section{References}

[1] O. Perron, "Die Stabilitätsfrage bei Differentialgleichungen," Mathematische Zeitschrift, vol. 32, no. 1, pp. 703-728, 1930.

[2] T. Li, "Die Stabilitätsfrage bei Differenzengleichungen," Acta Mathematica, vol. 63, no. 1, pp. 99-141, 1934.

[3] J. L. Massera and J. J. Schäffer, Linear Differential Equations and Function Spaces, vol. 21 of Pure and Applied Mathematics, Academic Press, New York, NY, USA, 1966.

[4] Ju. L. Daleckii and M. G. Krein, Stability of Solutions of Differential Equations in Banach Space, vol. 43 of Translations of Mathematical Monographs, American Mathematical Society, Providence, RI, USA, 1974.

[5] W. A. Coppel, Dichotomies in Stability Theory, Lecture Notes in Mathematics, Springer, Berlin, Germany, 1978.

[6] C. V. Coffman and J. J. Schäffer, "Dichotomies for linear difference equations," Mathematische Annalen, vol. 172, pp. 139166, 1967.

[7] D. Henry, Geometric Theory of Semilinear Parabolic Equations, vol. 840 of Lecture Notes in Mathematics, Springer, Berlin, Germany, 1981.

[8] R. P. Agarwal, Difference Equations and Inequalities: Theory, Methods and Applications, vol. 228 of Monographs and Textbooks in Pure and Applied Mathematics, Marcel Dekker, New York, NY, USA, 2nd edition, 2000.

[9] C. Pötzsche, Geometric Theory of Discrete Nonautonomous Dynamical Systems, vol. 2002 of Lecture Notes in Mathematics, Springer, Berlin, Germany, 2010.

[10] M. Pinto, "Discrete dichotomies," Computers \& Mathematics with Applications, vol. 28, no. 1-3, pp. 259-270, 1994.

[11] R. Naulin and M. Pinto, "Roughness of $(h, k)$-dichotomies," Journal of Differential Equations, vol. 118, no. 1, pp. 20-35, 1995.

[12] M. Megan, "On $(h, k)$-dichotomy of evolution operators in Banach spaces," Dynamic Systems and Applications, vol. 5, no. 2, pp. 189-196, 1996.

[13] J. L. Fenner, "On forward $(h, k)$ splitting for a class of noninvertible differential equations with impulse effect," Nonlinear Analysis: Theory, Methods \& Applications, vol. 67, no. 5, pp. 15131521, 2007.

[14] X. B. Lin, "Algebraic dichotomies with an application to the stability of Riemann solutions of conservation laws," Journal of Differential Equations, vol. 247, no. 11, pp. 2924-2965, 2009.

[15] A. J. G. Bento and C. M. Silva, "Generalized nonuniform dichotomies and local stable manifolds," http://arxiv.org/ abs/1007.5039.

[16] A. J. G. Bento and C. M. Silva, "Nonuniform $(\mu, \nu)$-dichotomies and local dynamics of difference equations," Nonlinear Analysis: Theory, Methods \& Applications, vol. 75, no. 1, pp. 78-90, 2012.

[17] A. J. G. Bento and C. M. Silva, "Nonuniform dichotomic behavior: Lipschitz invariant manifolds for difference equations," http://arxiv.org/abs/1209.6589.
[18] L. Barreira and C. Valls, "Lyapunov sequences for exponential dichotomies," Journal of Differential Equations, vol. 246, no. 1, pp. 183-215, 2009.

[19] L. Barreira, M. Fan, C. Valls, and J. Zhang, "Robustness of nonuniform polynomial dichotomies for difference equations," Topological Methods in Nonlinear Analysis, vol. 37, no. 2, pp. 357376, 2011.

[20] X. Chang, J. Zhang, and J. Qin, "Robustness of nonuniform $(\mu, \nu)$-dichotomies in Banach spaces," Journal of Mathematical Analysis and Applications, vol. 387, no. 2, pp. 582-594, 2012.

[21] J. Chu, "Robustness of nonuniform behavior for discrete dynamics," Bulletin des Sciences Mathématiques, 2013.

[22] Y. Jiang and F. F. Liao, "Admissibility for nonuniform $(\mu, \nu)$ contraction and dichotomy," Abstract and Applied Analysis, vol. 2012, Article ID 741696, 23 pages, 2012.

[23] J. Zhang, X. Chang, and J. Wang, "Existence and robustness of nonuniform $(h, k, \mu, v)$-dichotomies for nonautonomous impulsive differential equations," Journal of Mathematical Analysis and Applications, vol. 400, no. 2, pp. 710-723, 2013.

[24] J. M. Zhang, M. Fan, and X. Y. Chang, "Parameter dependence of stable manifolds for nonuniform $(\mu, v)$-dichotomies," Acta Mathematica Sinica (English Series), vol. 29, no. 6, pp. 1111-1130, 2013.

[25] B. Aulbach and J. Kalkbrenner, "Exponential forward splitting for noninvertible difference equations," Computers \& Mathematics with Applications, vol. 42, no. 3-5, pp. 743-754, 2001.

[26] L. Zhou, K. Lu, and W. Zhang, "Roughness of tempered exponential dichotomies for infinite-dimensional random difference equations," Journal of Differential Equations, vol. 254, no. 9, pp. 4024-4046, 2013.

[27] I. L. Popa, M. Megan, and T. Ceauşu, "Exponential dichotomies for linear discrete-time systems in Banach spaces," Applicable Analysis and Discrete Mathematics, vol. 6, no. 1, pp. 140-155, 2012.

[28] N. T. Huy and N. V. Minh, "Exponential dichotomy of difference equations and applications to evolution equations on the halfline," Computers \& Mathematics with Applications, vol. 42, no. 3-5, pp. 301-311, 2001.

[29] B. Sasu and A. L. Sasu, "On the dichotomic behavior of discrete dynamical systems on the half-line," Discrete and Continuous Dynamical Systems A, vol. 33, no. 7, pp. 3057-3084, 2013. 


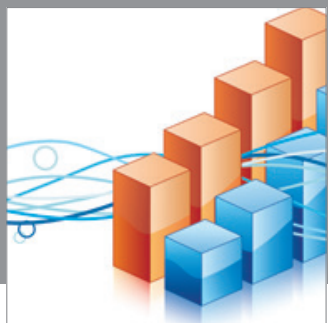

Advances in

Operations Research

mansans

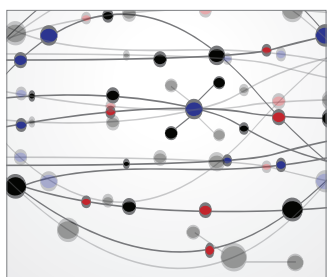

The Scientific World Journal
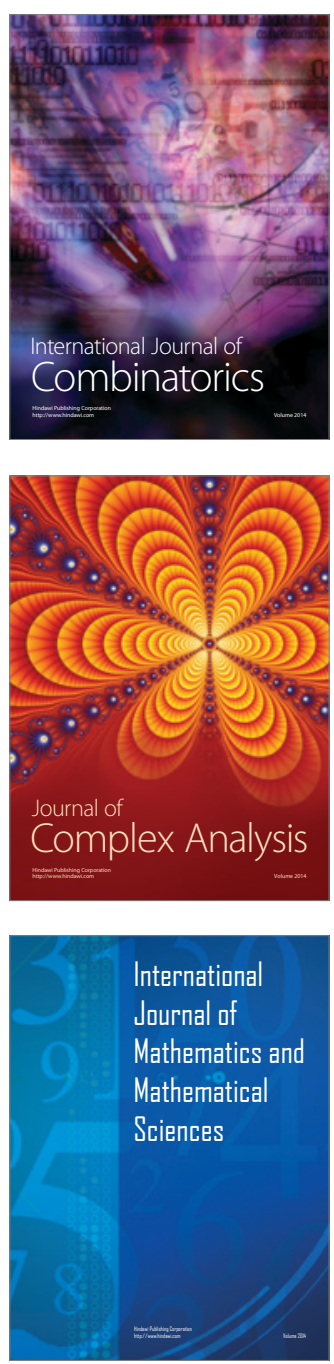
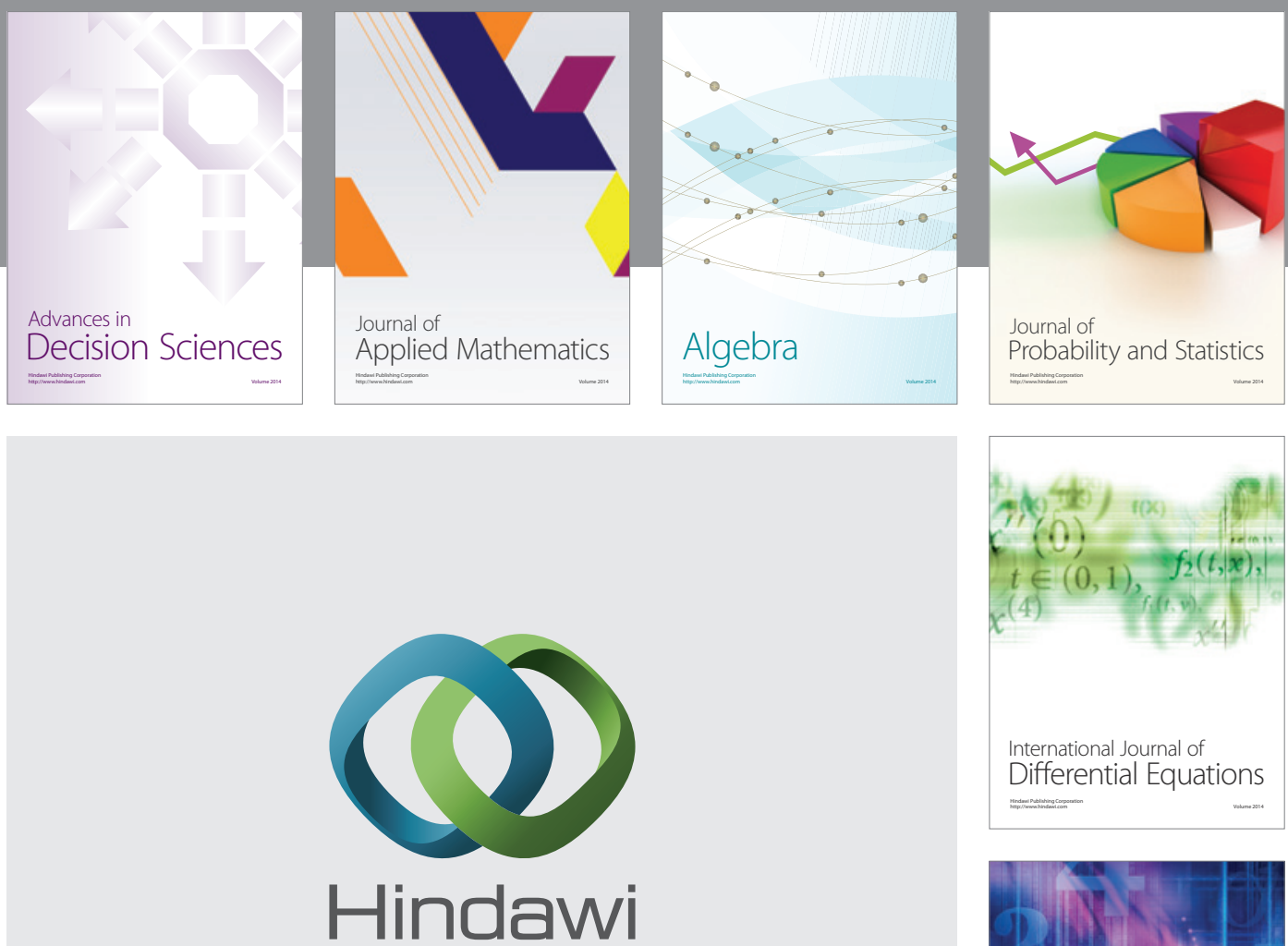

Submit your manuscripts at http://www.hindawi.com
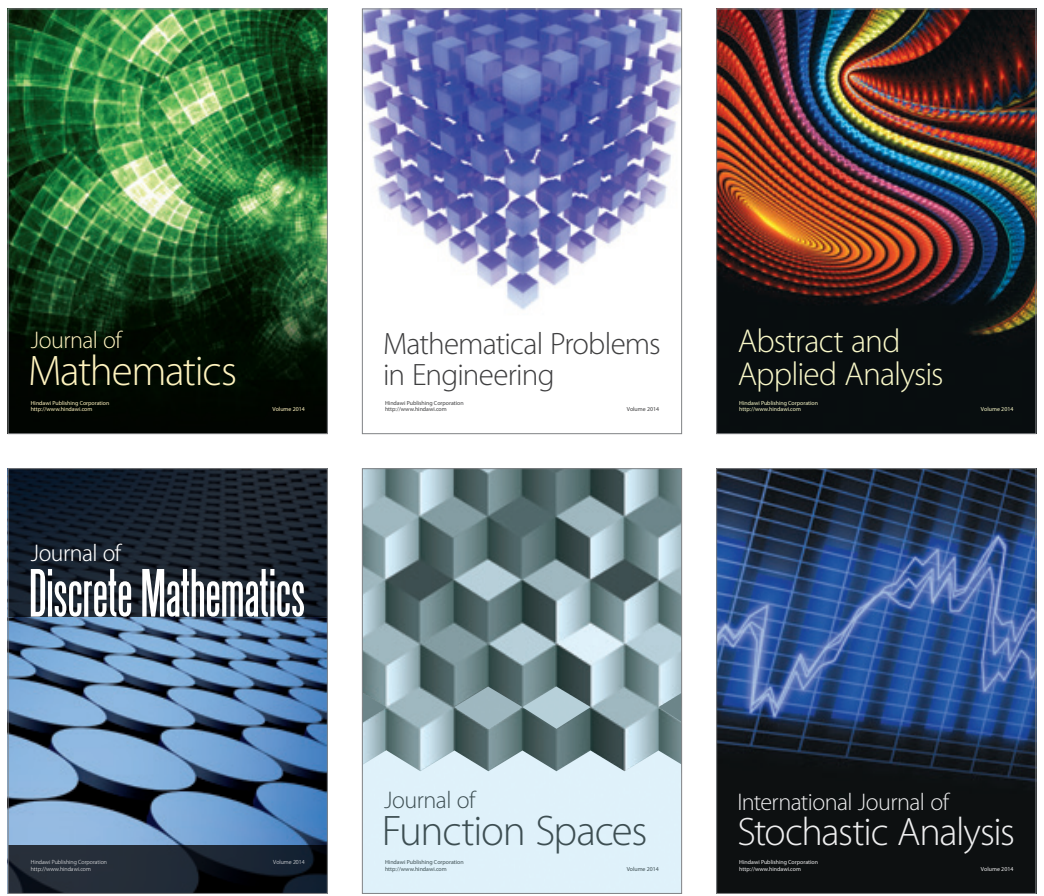

Journal of

Function Spaces

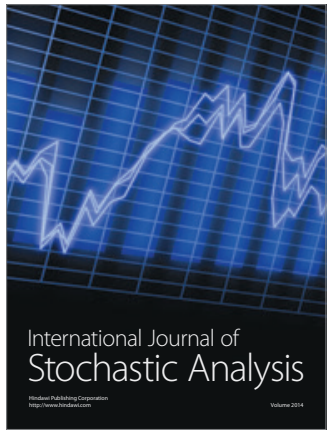

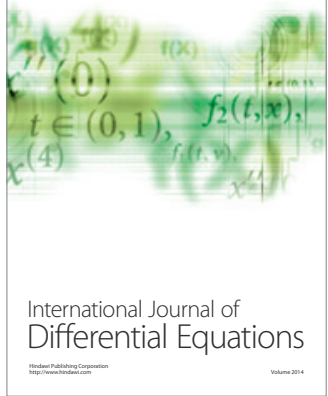
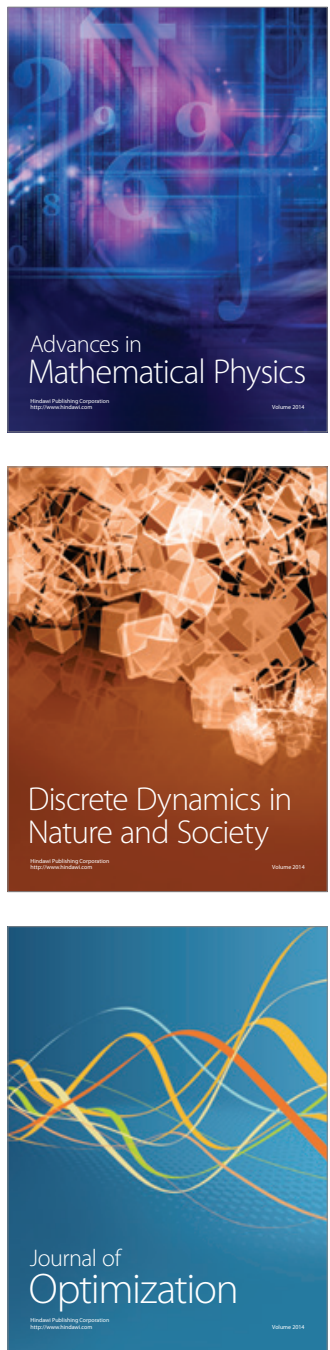